\title{
Model Predictive Control for Deflection-Limiting Maneuver of Flexible Structure
}

\author{
By Hirohisa KoJIMA ${ }^{1)}$ and Masataka TomiKawa ${ }^{2)}$ \\ ${ }^{1)}$ Department of Aerospace Engineering, Tokyo Metropolitan University, Tokyo, Japan \\ ${ }^{2)}$ Department of Aerospace Engineering, Tokyo Metropolitan Institute of Technology, Tokyo, Japan
}

(Received December 20th, 2007)

\begin{abstract}
An application of a model predictive control (MPC) for a deflection limiting maneuver of a flexible structure is studied. To limit the deflection of a flexible structure to within an allowable range during maneuver, deflection-limiting control (DLC) is employed as the primary control, and MPC is combined with DLC to overcome the disadvantages of DLC, including low robustness to modeling errors and disturbances. Moreover, a simple adaptive method is used to estimate the first modal frequency that dominates the vibration motion of the flexible structure. The effectiveness of the proposed algorithm is evaluated by numerical simulations.
\end{abstract}

Key Words: Model Predictive Control, Deflection-Limiting Control, Adaptive Method, Flexible Space Structure, Slew Maneuver

\section{Introduction}

Vibration of flexible space structures is undesirable when conducting precise attitude control. It is unlikely to be suppressed naturally once excited in space because of the vacuum of the space and the low structural damping characteristics of flexible space structures. Therefore, rest-to-rest motion is desired in maneuvering flexible space structures. Many control methods have been proposed to meet these objectives. Input shaping is one such control method, and there are various versions of input shaping control techniques, such as the Zero Vibration $(\mathrm{ZV}),{ }^{1,2)}$ the Zero Vibration and Derivative (ZVD), ${ }^{2)}$ the Specified-Insensitivity $(\mathrm{SI}),{ }^{3)}$ Extra-Insensitivity (EI) approach, ${ }^{4)}$ and minimum sensitivity control. ${ }^{5)}$ Adaptive input shaping control methods have been studied ${ }^{6-8)}$ to make the input shaping control more robust to the modeling errors. These adaptive input shaping methods, however, did not consider the deflection limiting of a flexible appendage. In order to limit deflection or a bending moment of the flexible appendage during maneuvers, the deflection-limiting control, ${ }^{9)}$ minimum bending-moment control ${ }^{10)}$ and its application to the multiobjective optimization control ${ }^{11)}$ have been proposed. The original deflection-liming control method, ${ }^{9)}$ however, was not robust to modeling errors, because it did not integrate an adaptive method. To overcome this disadvantage, an adaptive deflection-limiting control ${ }^{12)}$ has been proposed. However, the above input shaping control schemes can neither suppress the deflection nor achieve the desired maneuver angle or distance when significant external forces or torques disturb the system, because they are types of command shaping control.

To overcome the above disadvantage of input shaping schemes, feedback type control methods should be combined with input shaping control methods. For this purpose, feedback control such as PD control, ${ }^{13)}$ PID control, ${ }^{14)}$ slid-

(C) 2009 The Japan Society for Aeronautical and Space Sciences ing-mode control, ${ }^{15)}$ and $H_{\infty}$ control $^{16)}$ have been combined with input shaping. However, deflection limiting has still not been considered in these studies. In this study, a model predictive control technique is applied to deflection-limiting control as a feedback control to limit the bendingmoment at the root of a flexible beam within a specific value during a slewing maneuver. This method works even when there are modeling errors and external disturbances. In the proposed control scheme, a reference control profile resulting from the deflection-limiting control is used, and the first modal frequency that is the dominant effect for the vibration motion of the flexible beam is estimated by taking into account the relationships between the bending-moment of the beam and the control input. The deviation of the maneuver angle is used as one of the state values to determine the model predictive control input to compensate for the modeling errors and external disturbances. The validity of the proposed control scheme is verified by numerical simulations. Section 2 describes the system model of the flexible structure. Section 3 explains the control schemes, and the results of numerical simulations to demonstrate the effectiveness of the proposed method. Section 4 summarizes the conclusion of this paper.

\section{System Model}

\subsection{Nominal model}

Figure 1 shows a model of a space structure consisting of a rigid body and flexible appendage. The appendage is a cantilever beam with one end fixed to the rigid body and the other end free. The rotational and vibrational motion is assumed to be two-dimensional and planar. Control torque is applied to the center of the rigid body. In the present analysis, structural damping and the air drag are neglected for simplicity, because most space structures are very lightly damped. $I_{\mathrm{r}}$ is the moment of inertia, $L$ is the radius of the main body, $l$ is the length of the beam, and $E I$ is the rigidity 


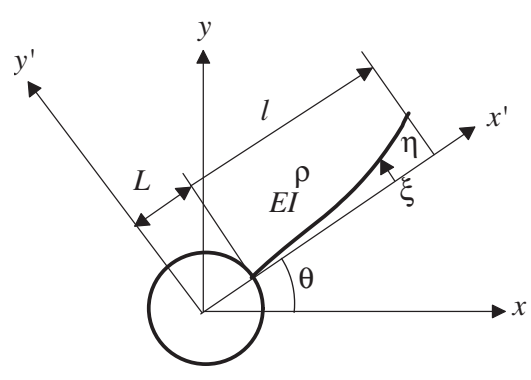

Fig. 1. Model of flexible structure.

of the beam. Both $l$ and $E I$ are assumed to be constant along the flexible appendage. The displacement of the beam is denoted by $\eta$ at the position of $\xi$, as shown in Fig. 1 .

By considering the following boundary conditions

$$
\begin{gathered}
\eta=\frac{\partial \eta}{\partial \xi}=0 \quad \text { at } \quad \xi=L, \\
\frac{\partial^{2} \eta}{\partial \xi^{2}}=\frac{\partial^{3} \eta}{\partial \xi^{3}}=0 \quad \text { at } \quad \xi=L+l,
\end{gathered}
$$

expanding the flexible vibration of the appendage using vibrational modes that can be described as

$$
\eta(\xi, t)=\sum \phi_{i}(\xi) q_{i}(t)
$$

and assuming the orthogonal condition that vibrational mode variables satisfy:

$$
\begin{aligned}
& \frac{\partial^{4} \phi_{i}}{\partial \xi^{4}}-\beta_{i}{ }^{4} \phi_{i}=0, \\
& \int_{L}^{L+l} \phi_{i} \phi_{j} \mathrm{~d} \xi=\delta_{i j},
\end{aligned}
$$

where $\delta_{i j}$ is the Kronecker delta, the equations of motion can be written in the form of the linear time invariant (LTI) system as

$$
\begin{aligned}
& I_{\mathrm{r}} \ddot{\theta}-E I \sum_{i=1}^{n} \alpha_{i} \beta_{i}{ }^{4} q_{i}=T, \\
& E I \beta_{i}{ }^{4} q_{i}+\rho\left(\alpha_{i} \ddot{\theta}+\ddot{q}_{i}\right)=0,
\end{aligned}
$$

where $\alpha_{i}$ and $\beta_{i}$ are coefficients determined from the system parameters. These equations can be rewritten as

$$
\boldsymbol{M} \ddot{\boldsymbol{q}}+\boldsymbol{K} \boldsymbol{q}=\boldsymbol{Q} u,
$$

where $\boldsymbol{M}$ and $\boldsymbol{K}$ are the mass and rigidity matrix, respectively, $\boldsymbol{q}$ is a state vector, and $u$ is the control input $(T)$. Considering the eigenvalues of the system, Eq. (8) can be rewritten in state vector form as

$$
\dot{\boldsymbol{x}}=\boldsymbol{A}_{\mathrm{c}} \boldsymbol{x}+\boldsymbol{B}_{\mathrm{c}} u,
$$

where matrices $\boldsymbol{A}_{\mathrm{c}}$ and $\boldsymbol{B}_{\mathrm{c}}$ are given as

$$
\begin{gathered}
\boldsymbol{A}_{\mathrm{c}}=\text { blockdiag }\left[\begin{array}{cc}
0 & 1 \\
-\omega_{i}^{2} & 0
\end{array}\right], \\
(i=0,1,2, \cdots, n) \\
\boldsymbol{B}_{\mathrm{c}}=\left[\begin{array}{lllllll}
0 & b_{0} & 0 & b_{1} & \cdots & 0 & b_{n}
\end{array}\right]^{\mathrm{T}},
\end{gathered}
$$

and $\omega_{i}$ and $b_{i}$ are the $i$ th modal frequency and the $i$ th modal
Table 1. System parameters.

\begin{tabular}{ll}
\hline Length of beam $l$ & $0.845 \mathrm{~m}$ \\
Rigidity $E I$ & $0.175 \mathrm{~N} \mathrm{~m}^{2}$ \\
Mass density $\rho$ & $9.16 \times 10^{-2} \mathrm{~kg} / \mathrm{m}$ \\
Moment of inertia $I_{\mathrm{r}}$ & $0.14 \mathrm{~kg} \mathrm{~m}^{2}$ \\
Radius $L$ & $0.0338 \mathrm{~m}$ \\
Maximum torque & $0.15 \mathrm{Nm}$ \\
Slew angle $\theta_{\mathrm{f}}$ & $60 \mathrm{deg}$ \\
Deflection limit & $0.01 \mathrm{Nm}$ \\
\hline
\end{tabular}

gain of the system, respectively. Note that the modal frequencies $\omega_{i}$ are not equivalent to the natural frequencies of the beam alone.

The bending moment at the root of the beam, $B_{\mathrm{e}}$, is represented using the state variables as

$$
B_{\mathrm{e}}=\boldsymbol{C x}
$$

where $\boldsymbol{C}$ is the coefficient matrix related to the deflection of the modal vibration. If the vibrational motion can be mainly represented by the first mode, the equation of motion can be reduced to

$$
\frac{\mathrm{d}}{\mathrm{d} t}\left[\begin{array}{c}
x_{1} \\
\dot{x}_{1} \\
x_{2} \\
\dot{x}_{2}
\end{array}\right]=\left[\begin{array}{cccc}
0 & 1 & 0 & 0 \\
0 & 0 & 0 & 0 \\
0 & 0 & 0 & 1 \\
0 & 0 & -\omega_{1}^{2} & 0
\end{array}\right]\left[\begin{array}{c}
x_{1} \\
\dot{x}_{1} \\
x_{2} \\
\dot{x}_{2}
\end{array}\right]+\left[\begin{array}{c}
0 \\
b_{1} \\
0 \\
b_{2}
\end{array}\right] u,
$$

and the bending moment at the root of the beam is represented simply as

$$
B_{\mathrm{e}}=C_{2} x_{2}
$$

Thus, the bending-moment satisfies

$$
\ddot{B_{\mathrm{e}}}+\omega_{1}^{2} B_{\mathrm{e}}=C_{2} b_{2} u .
$$

When the bending-moment is small, the angular acceleration of the main body is given by

$$
\ddot{\theta}=C_{1} b_{1} u \text {. }
$$

The parameters of the nominal model are listed in Table 1. Note that using these parameters, the first modal frequency is determined analytically as $7.27 \mathrm{rad} / \mathrm{s}$.

\subsection{External disturbance}

It is almost impossible to achieve a physical system that is unaffected by external disturbances. In this study, it is assumed that the system model is in lean air and that the airdrag force represented as

$$
F(\xi)=k[\xi \dot{\theta}+\dot{q} \phi(\xi)]
$$

affects the nominal model as an external disturbance. In this case, the right-hand term in Eq. (6) should be replaced with

$$
T^{\prime}=T-\int_{L}^{L+l} F(\xi) \xi \mathrm{d} \xi,
$$

and the right-hand term in Eq. (7) becomes

$$
Q_{q}=-\int_{L}^{L+l} F(\xi) \phi(\xi) \mathrm{d} \xi .
$$




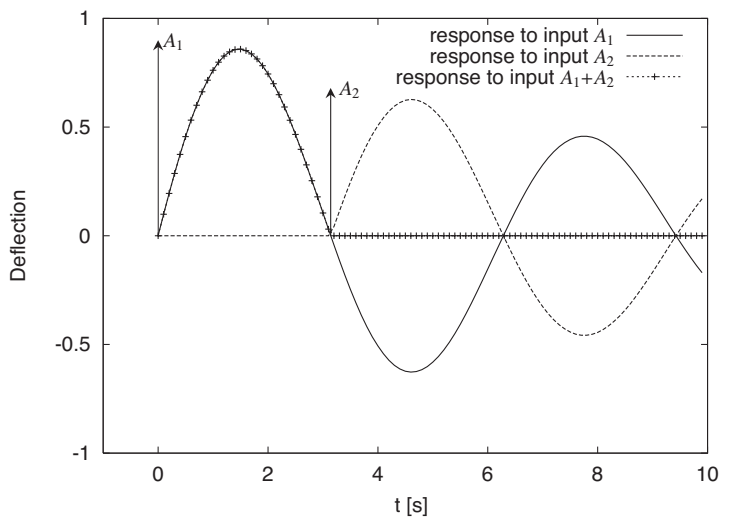

Fig. 2. Basic concept of input shaping.

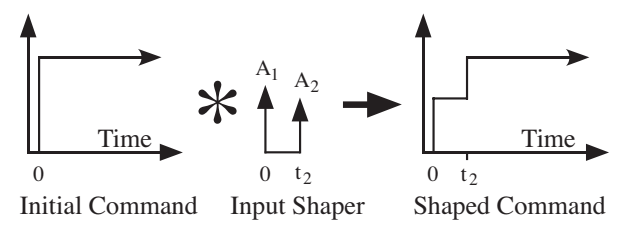

Fig. 3. Convolution with input shaping.

\section{Control Laws}

The objective of this study is to derive a control law that can achieve the desired maneuver angle of $60 \mathrm{deg}$ and to limit the bending-moment at the root of the beam to within $0.01 \mathrm{~N} \mathrm{~m}$ during maneuver, even if there is a modeling error in the mathematical model and external disturbances affect the system. In this section, the basic concept of input shaping and deflection-limiting control based on the input shaping is explained. Then, the deflection-liming control in conjunction with model predictive control is addressed.

\subsection{Input shaping}

Input shaping is a control method that uses a sequence of impulses as the representative form of the control input to cancel the residual vibration. Figure 2 shows a schematic representation of the $\mathrm{ZV}$ control, in which the second impulsive control input is given as the half period of the vibration. In addition, an input shaper can be convolved with any control input to generate a properly shaped command, as shown in Fig. 3.

Figure 4 shows the results of a bang-bang control and a ZV control when applied to a nominal system model without modeling errors and external disturbance described in section 2. Bang-bang control induces large vibrations, but ZV control does not. However, ZV control does not limit the bending-moment within the restricted range.

\subsection{Deflection-limiting control}

When the allowable bending-moment is limited within some values, simple input shaping methods may not be appropriate. To satisfy the constraints on deflection or bending-moment during maneuver, deflection-limiting control (DLC) has been proposed. ${ }^{9)}$ Figure 5 shows DLC.

The command switch timings and the magnitude of the DLC are determined using the first modal frequency, $\omega_{1}$,

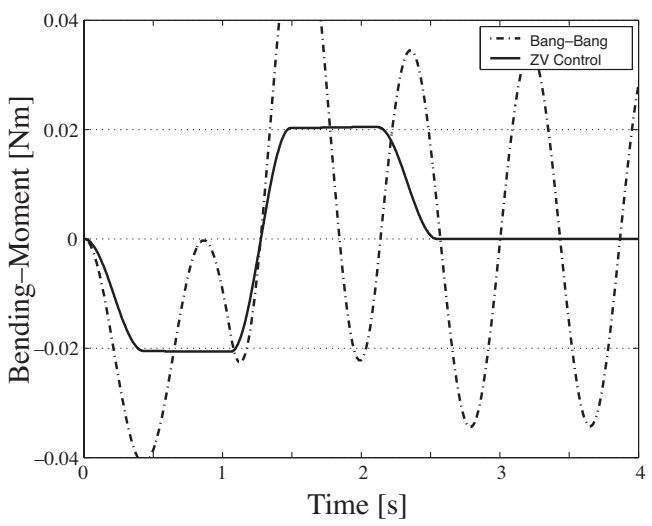

Fig. 4. Results of bang-bang control and input shaping.

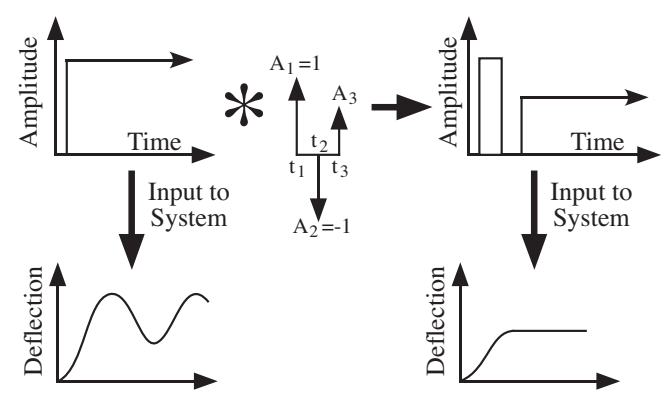

Fig. 5. Deflection-limiting control(DLC).

and the acceptable deflection as follows:

$$
\begin{gathered}
t_{2}=\frac{1}{\omega_{1}} \cos ^{-1}\left(1-2 D L^{2}\right), \\
t_{3}=\frac{1}{\omega_{1}} \cos ^{-1}(-D L), \\
A_{3}=2 D L, \quad D L=\frac{D_{\text {limit }}}{D_{\max }},
\end{gathered}
$$

where $D_{\max }$ is the maximum magnitude of the vibration induced by the maximum step input. Figure 6 shows the time response of the bending-moment driven by DLC for the cases with and without modeling error.

Because the control switching times and magnitude of control input depend on the first modal frequency, DLC cannot be guaranteed to limit the bending moment within a specific value when the modeled frequency differs from the actual frequency, as shown in Fig. 6.

\subsection{Model predictive control}

\subsubsection{Concept}

Model predictive control (MPC) methods ${ }^{17)}$ are an optimal feedback control method in a sense that control inputs are given to the system so that the difference between the predicted state variables and the reference state variables is minimized in the specified sampling period in the future, and can take into account the constraints on input magnitude as well as output magnitude. A schematic representation of MPC is shown in Fig. 7.

The current plant state is sampled at time $t$, and control inputs minimizing cost for a short time horizon in the future: $[t, t+p \Delta]$ are computed, where $p$ is the horizon steps. State 


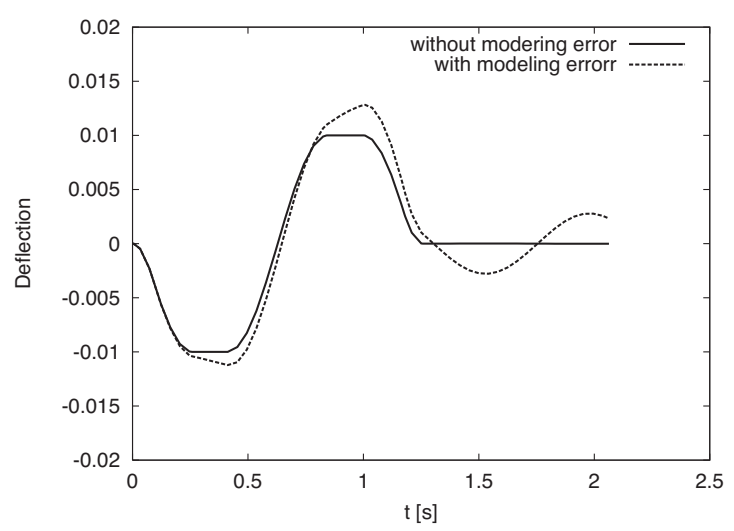

Fig. 6. Robustness of DLC.

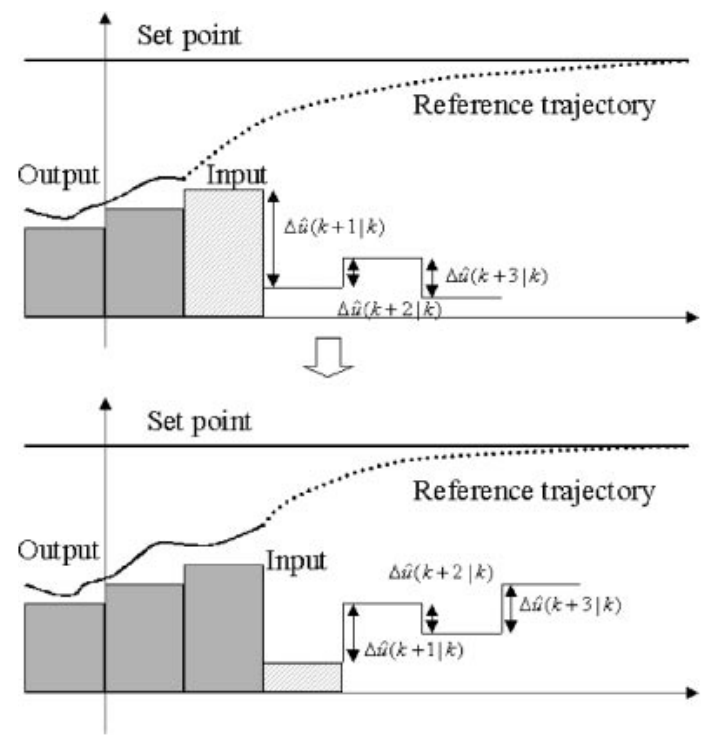

Fig. 7. Basic principle of model predictive control (MPC).

trajectories are predicted from the current state by applying the control inputs to the mathematical plant model. Only the first step of the control inputs is implemented to the plant. Then, the plant state is sampled at the next sampling time again and this calculation is repeated. If a mathematical model is a linear time invariant (LTI) system, and a cost function is given in the form of a quadratic regulator problem, the optimal control gain is obtained in advance. That is, the cost minimization problem does not need to be solved with respect to control input at every sampling time.

The following subsection shows how to determine the optimal control variation $\Delta \hat{u}(k \mid k)_{\text {opt }}$ in MPC with constraints.

\subsubsection{Deviation of optimal control input}

From the equation of motion, the predicted state variables are obtained as

$$
\begin{aligned}
\hat{\boldsymbol{x}}(k+1 \mid k)= & \boldsymbol{A} \hat{\boldsymbol{x}}(k)+\boldsymbol{B} \hat{u}(k) \\
= & \hat{\boldsymbol{A}} \boldsymbol{x}(k) \\
& +\hat{\boldsymbol{B}}\left[\hat{u}(k-1)+\left\{u_{\mathrm{d}}(k)-u_{\mathrm{d}}(k-1)\right\}\right. \\
& +\Delta \hat{\boldsymbol{u}}(k \mid k)],
\end{aligned}
$$

$$
\begin{aligned}
\hat{\boldsymbol{x}}(k+2 \mid k)= & \boldsymbol{A} \hat{\boldsymbol{x}}(k+1 \mid k)+\boldsymbol{B} \hat{u}(k+1) \\
= & \boldsymbol{A} \hat{\boldsymbol{x}}(k+1 \mid k) \\
& +\boldsymbol{B}\left[\hat{u}(k)+\boldsymbol{B}\left\{u_{\mathrm{d}}(k+1)-u_{\mathrm{d}}(k)\right\}\right. \\
& +\Delta \hat{\boldsymbol{u}}(k+1 \mid k)], \\
\hat{\boldsymbol{x}}(k+3 \mid k)= & \cdots,
\end{aligned}
$$

where $\Delta \hat{u}(k+i \mid k)$ is the control input deviation at the predictive time step, matrices $\boldsymbol{A}$ and $\boldsymbol{B}$ are given as

$$
\begin{aligned}
& \boldsymbol{A}=e^{\boldsymbol{A}_{\mathrm{c}} \Delta}, \\
& \boldsymbol{B}=\int_{0}^{\Delta} e^{\boldsymbol{A}_{\mathrm{c}} \tau} \boldsymbol{B}_{\mathrm{c}} \mathrm{d} \tau=\left(e^{\boldsymbol{A}_{\mathrm{c}} \Delta}-\boldsymbol{I}_{4}\right) \boldsymbol{A}_{\mathrm{c}}{ }^{-1} \boldsymbol{B}_{\mathrm{c}},
\end{aligned}
$$

and $u_{\mathrm{d}}$ denotes the control input determined by DLC.

In this study, the state variables predicted in four steps are used for control. The optimal control deviation $\Delta \hat{u}(k \mid k)_{\text {opt }}$ is determined so that the following performance index is minimized.

$$
J=\int_{t}^{t+5 \Delta}\left(z^{\mathrm{T}}(t) \boldsymbol{Q} z(t)+\Delta u^{\mathrm{T}}(t) R \Delta u(t)\right) \mathrm{d} t,
$$

where $\boldsymbol{z}(t)=\boldsymbol{x}(t)-\boldsymbol{r}(t)$, and $\boldsymbol{Q}$ and $R$ are weighting matrices. Discretizing the above equation yields

$$
\begin{aligned}
J= & \sum_{i=1}^{5}\|\hat{\boldsymbol{x}}(k+i \mid k)-\boldsymbol{r}(k+i \mid k)\|_{\boldsymbol{Q}}^{2} \\
& +\sum_{i=0}^{4}\|\Delta \hat{u}(k+i \mid k)\|_{R}^{2},
\end{aligned}
$$

where $\boldsymbol{r}(k+i \mid k)$ is the reference output at the time node of $k+i$.

Introducing

$$
\begin{aligned}
\boldsymbol{X}(k)= & {\left[\begin{array}{lll}
\hat{\boldsymbol{x}}(k+1 \mid k)^{\mathrm{T}} & \cdots & \hat{\boldsymbol{x}}(k+5 \mid k)^{\mathrm{T}}
\end{array}\right]^{\mathrm{T}}, } \\
\boldsymbol{T}(k)= & {\left[\begin{array}{lll}
\boldsymbol{r}(k+1 \mid k)^{\mathrm{T}} & \cdots & \boldsymbol{r}(k+5 \mid k)^{\mathrm{T}}
\end{array}\right]^{\mathrm{T}}, } \\
\Delta \boldsymbol{U}(k)= & {\left[\begin{array}{lll}
\Delta \hat{u}(k \mid k) & \cdots & \Delta \hat{\boldsymbol{u}}(k+4 \mid k)
\end{array}\right]^{\mathrm{T}}, } \\
\Delta \boldsymbol{U}_{\mathrm{d}}(k)= & {\left[\begin{array}{c}
u_{\mathrm{d}}(k)-u_{\mathrm{d}}(k-1) \\
u_{\mathrm{d}}(k+1)-u_{\mathrm{d}}(k) \\
u_{\mathrm{d}}(k+2)-u_{\mathrm{d}}(k+1) \\
u_{\mathrm{d}}(k+3)-u_{\mathrm{d}}(k+2) \\
u_{\mathrm{d}}(k+4)-u_{\mathrm{d}}(k+3)
\end{array}\right], }
\end{aligned}
$$

Equations (23) and (24) can then be rewritten as

$$
\boldsymbol{X}(k)=\boldsymbol{\Phi} \boldsymbol{x}(k)+\boldsymbol{\Upsilon} \hat{u}(k-1)+\boldsymbol{\Theta} \Delta \boldsymbol{U}(k)+\boldsymbol{\Theta}^{\prime} \Delta \boldsymbol{U}_{\mathrm{d}}(k),
$$

where

$$
\boldsymbol{\Phi}=\left[\begin{array}{c}
\boldsymbol{A} \\
\boldsymbol{A}^{2} \\
\cdots \\
\boldsymbol{A}^{5}
\end{array}\right], \quad \boldsymbol{\Upsilon}=\left[\begin{array}{c}
\boldsymbol{B} \\
\sum_{i=0}^{1} \boldsymbol{A}^{i} \boldsymbol{B} \\
\cdots \\
\sum_{i=0}^{4} \boldsymbol{A}^{i} \boldsymbol{B}
\end{array}\right],
$$




$$
\begin{aligned}
\boldsymbol{\Theta} & =\left[\begin{array}{cccc}
\boldsymbol{B} & \mathbf{0} & \mathbf{0} & \mathbf{0} \\
\boldsymbol{A} \boldsymbol{B}+\boldsymbol{B} & \boldsymbol{B} & \mathbf{0} & \mathbf{0} \\
\vdots & \ddots & \ldots & \mathbf{0} \\
\sum_{i=0}^{4} \boldsymbol{A}^{i} \boldsymbol{B} & \sum_{i=0}^{3} \boldsymbol{A}^{i} \boldsymbol{B} & \ldots & \boldsymbol{B}
\end{array}\right], \\
\boldsymbol{\Theta}^{\prime} & =\left[\begin{array}{ccccc}
\boldsymbol{B} & \mathbf{0} & \mathbf{0} & \mathbf{0} & \mathbf{0} \\
\boldsymbol{A} \boldsymbol{B}+\boldsymbol{B} & \boldsymbol{B} & \mathbf{0} & \mathbf{0} & \mathbf{0} \\
\vdots & \ddots & \ldots & \ldots & \mathbf{0} \\
\sum_{i=0}^{4} \boldsymbol{A}^{i} \boldsymbol{B} & \sum_{i=0}^{3} \boldsymbol{A}^{i} \boldsymbol{B} & \ldots & \boldsymbol{A} \boldsymbol{B} & \boldsymbol{B}
\end{array}\right] .
\end{aligned}
$$

In addition,

$$
\begin{gathered}
\boldsymbol{Q}^{\prime}=\operatorname{blockdiag}[\boldsymbol{Q}, \boldsymbol{Q}, \boldsymbol{Q}, \boldsymbol{Q}, \boldsymbol{Q}], \\
\boldsymbol{R}=\operatorname{diag}[R, R, R, R, R] .
\end{gathered}
$$

The performance index Eq. (28) can then be reduced to

$$
J=\text { const. }+\Delta \boldsymbol{U}(k)^{\mathrm{T}} \boldsymbol{G}(k)+\frac{1}{2} \Delta \boldsymbol{U}(k)^{\mathrm{T}} \boldsymbol{H} \Delta \boldsymbol{U}(k)
$$

where

$$
\begin{aligned}
\boldsymbol{G}(k)= & -2 \boldsymbol{\Theta}^{\mathrm{T}} \boldsymbol{Q}^{\prime} \boldsymbol{E}(k), \\
\boldsymbol{H}= & 2\left[\frac{1}{2} \boldsymbol{\Theta}^{\mathrm{T}} \boldsymbol{Q}^{\prime} \boldsymbol{\Theta}+\boldsymbol{R}\right], \\
\boldsymbol{E}(k)= & \boldsymbol{T}(k)-[\boldsymbol{\Phi} \boldsymbol{x}(k)+\boldsymbol{\Upsilon} u(k-1)] \\
& -\boldsymbol{\Theta}^{\prime} \Delta \boldsymbol{U}_{\mathrm{d}}(k) .
\end{aligned}
$$

If the constraints of dimension $n$ are given with respect to the state variables, then the constraints can be converted with respect to the control input variations as

$$
\boldsymbol{\Omega} \Delta \boldsymbol{U}(k) \leq \boldsymbol{\Psi},
$$

where $\boldsymbol{\Omega}$ is a matrix of dimension $n \times 5$, and $\boldsymbol{\Psi}$ is a vector representing the constraints. This study introduces the constraint such that the bending-moment in four steps is less than $0.01 \mathrm{Nm}$.

$$
\left\|B_{\mathrm{e}}(k+5 \mid k)\right\| \leq 0.01
$$

Recalling Eq. (12), Eq. (44) is rewritten as

$$
\left\|C_{2} \hat{x}_{2}(k+5 \mid k)\right\| \leq 0.01 \text {. }
$$

The problem of obtaining the optimal control deviation $\Delta \boldsymbol{U}(k)$ is equivalent to the following optimal control problem with constraints

$$
\begin{aligned}
& \min \left[\frac{1}{2} \Delta \boldsymbol{U}(k)^{\mathrm{T}} \boldsymbol{H} \Delta \boldsymbol{U}(k)+\boldsymbol{G}(k) \Delta \boldsymbol{U}(k)\right] \\
& \text { subject to } \boldsymbol{\Omega} \Delta \boldsymbol{U}(k) \leq \psi .
\end{aligned}
$$

When the constraint is inactive, the optimal control deviation $\Delta \hat{u}(k \mid k)_{\text {opt }}$ is obtained by considering the gradient of the performance index with respect to the control deviation as follows:

$$
\Delta \boldsymbol{U}(k)=-\boldsymbol{H}^{-1} \boldsymbol{G}(k) .
$$

On the other hand, if the above control deviation $\Delta \boldsymbol{U}(k)$ does not satisfy the inequality constraint, Eq. (39) can be simply represented as

$$
\begin{aligned}
& \min \left[\boldsymbol{G}(k)^{\mathrm{T}} \Delta \boldsymbol{U}(k)+\frac{1}{2} \Delta \boldsymbol{U}(k)^{\mathrm{T}} \boldsymbol{H} \Delta \boldsymbol{U}(k)\right] \\
& \text { subject to } \boldsymbol{\Omega} \Delta \boldsymbol{U}(k)=\psi .
\end{aligned}
$$

Introducing a Lagrange multiplier $\lambda$, Eq. (48) can be rewritten as

$$
\left[\begin{array}{c}
\Delta \boldsymbol{U}(k) \\
\lambda
\end{array}\right]=\left[\begin{array}{cc}
\boldsymbol{H} & \boldsymbol{\Omega}^{\mathrm{T}} \\
\boldsymbol{\Omega} & 0
\end{array}\right]^{-1}\left[\begin{array}{c}
-\boldsymbol{G}(k)^{\mathrm{T}} \\
\psi
\end{array}\right] .
$$

Consequently, the optimal control deviation is given by

$$
\Delta \hat{u}(k \mid k)_{\mathrm{opt}}=\left[\begin{array}{lllll}
1 & 0 & 0 & 0 & 0
\end{array}\right] \Delta \boldsymbol{U}(k),
$$

if $P \leq \psi$ then

$$
\Delta \boldsymbol{U}(k)=-\boldsymbol{H}^{-1} \boldsymbol{G}(k)
$$

else

$$
\Delta \boldsymbol{U}(k)=\left[\begin{array}{ll}
\boldsymbol{I}_{4} & \mathbf{0}
\end{array}\right]\left[\begin{array}{cc}
\boldsymbol{H} & \boldsymbol{\Omega}^{\mathrm{T}} \\
\boldsymbol{\Omega} & \mathbf{0}
\end{array}\right]^{-1}\left[\begin{array}{c}
-\boldsymbol{G}(k) \\
\psi
\end{array}\right]
$$

where

$$
P=-\boldsymbol{\Omega} \boldsymbol{H}^{-1} \boldsymbol{G}(k)
$$

\subsection{Deflection-limiting control incorporating model predictive control}

The objective of this study is to apply MPC to DLC as the feedback control so that the following four conditions are satisfied, even if the actual first modal frequency is 1.3 times the analytically predicted modal frequency, which is $7.27 \mathrm{rad} / \mathrm{s}$.

- The desired slew angle of $60 \mathrm{deg}$ is achieved.

- The angular velocity is zero at the end of the slewing maneuver.

- Residual vibration is suppressed.

- The deflection is limited to within the acceptable level (The bending-moment is within $0.01 \mathrm{~N} \mathrm{~m}$.)

In this study, three control schemes are studied under the conditions of weighting parameters $Q=$ $\operatorname{diag}[1,0,1000,0]$ and $R=10^{-4}$, sampling time $\Delta=$ $0.003 \mathrm{~s}$, and disturbance coefficient $k=10^{-3}$.

\subsubsection{Control law A}

Control law A consists of the following two steps:

1. First, the control profile of the DLC is determined using the predicted first modal frequency. The time response of the state variable is predicted using this control profile when there are no modeling errors, and the predicted time response of the state variables is set to the reference trajectories.

2. Second, the optimal control variation is determined so that the bending-moment is limited to within an acceptable level, and the state variables track the trajectories predicted in step-1. Then, the control input is applied to the system. 

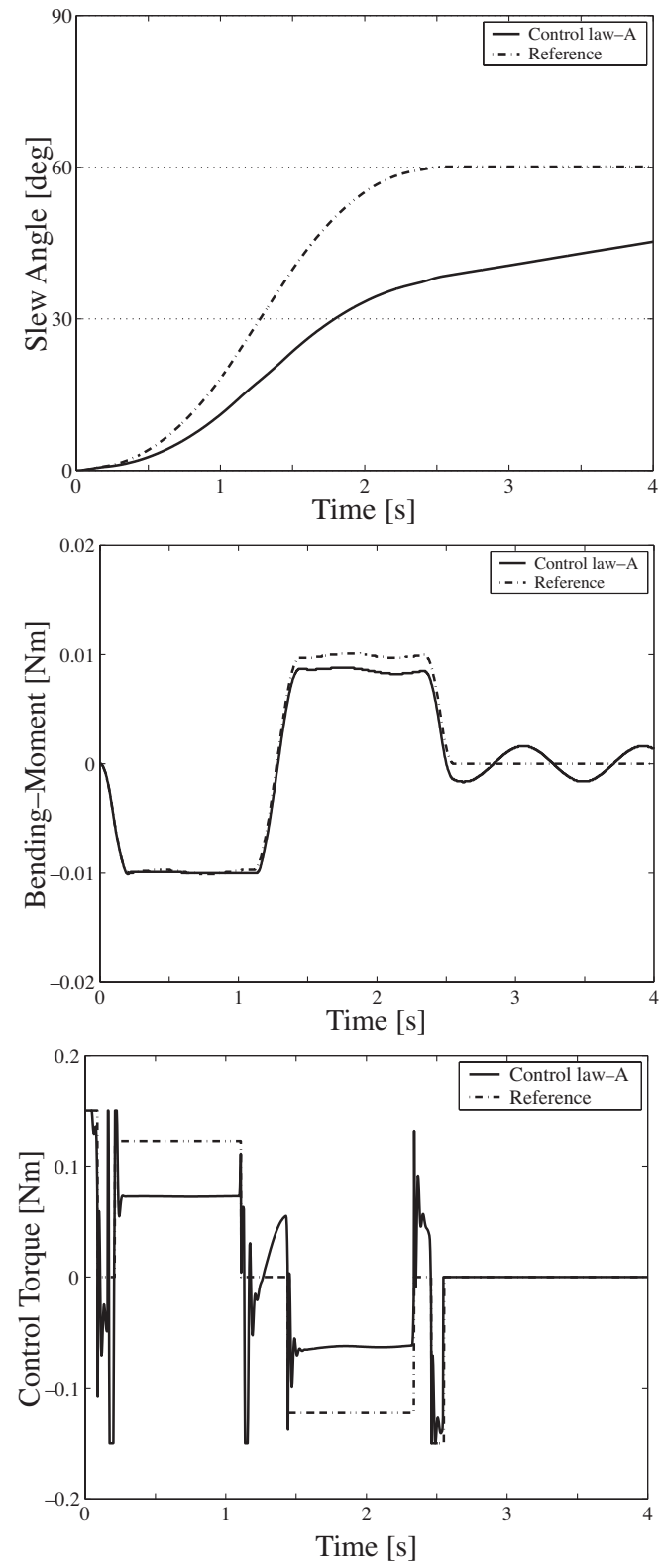

Fig. 8. Results of control law A.

The time responses of the bending-moment, slewing angle and control input resulting from the numerical simulations of control law A are shown in Fig. 8. The reference trajectories are also shown in this figure to demonstrate the effectiveness of MPC.

As shown in Fig. 8, the bending-moment resulting from MPC is almost within the acceptable range. However, the control input becomes asymmetric with respect to the middle of the maneuver due to the feedback control input, which results in the bias angular velocity at the end of maneuver, that is, the slewing angle does not converge. In addition, the desired slewing angle is not achieved, and some residual vibration remains at the end of control input.

\subsubsection{Control law B}

To make the slewing angle converge at the end of control input, the control input profile must be symmetric with respect to half of the control duration $\left(T_{\mathrm{m}}\right)$. To implement
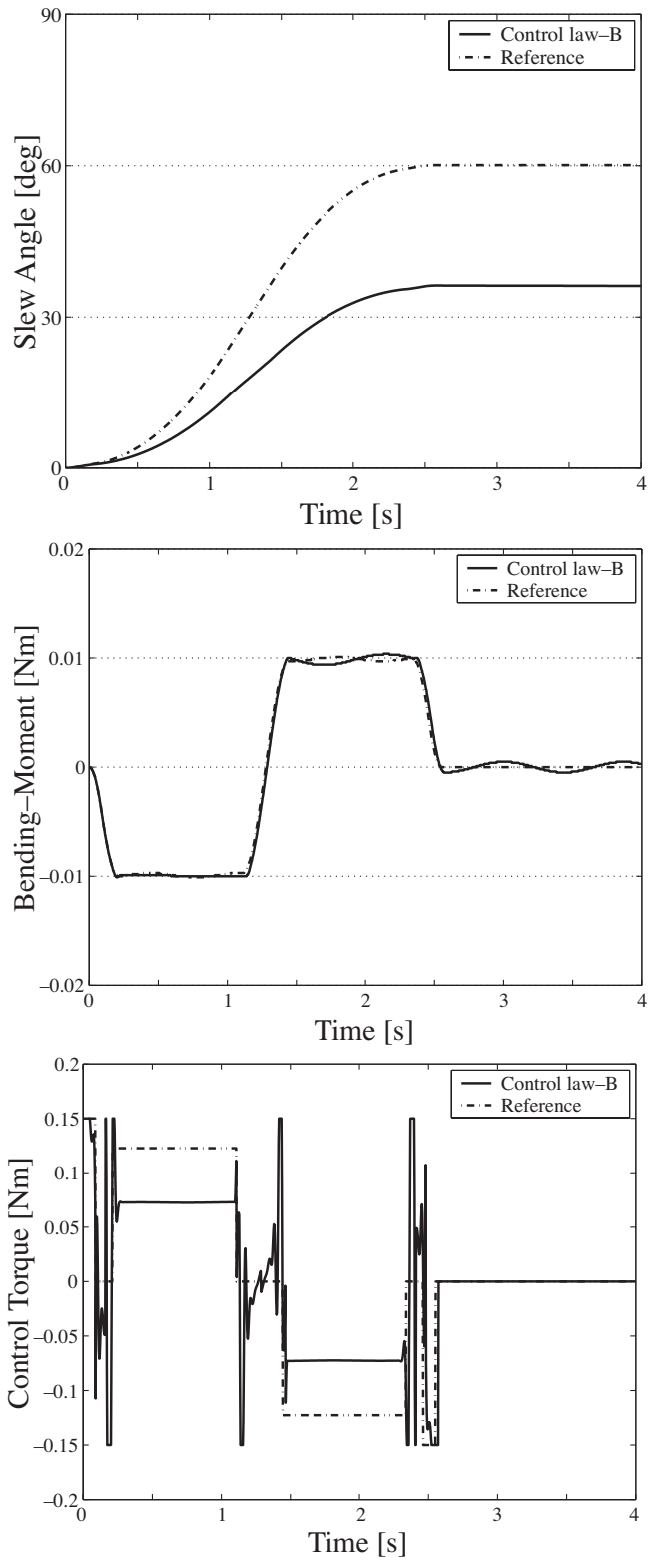

Fig. 9. Results of control law B.

this profile, feedback control by MPC is used until $T_{\mathrm{m}}$, and the control input after $T_{\mathrm{m}}$ is made symmetric with respect to $T_{\mathrm{m}}$, instead of being determined by MPC. The control profile determined by this approach is called control law B. The results of numerical simulations for control law B are shown in Fig. 9.

As a result of symmetric control profile, both the residual vibrations and angular velocity become zero at the end of control input. However, the desired slewing angle is still not achieved, because the condition to limit the bendingmoment within the specified value was given higher priority than the slewing angle, and the difference between the estimated first modal frequency and the actual modal frequency exceeded the predicted range.

To overcome this problem, the first modal frequency in the mathematical model should be tuned during the maneuver. 
Next, we consider control law $\mathrm{C}$, which is a control method with an adaptive law to tune the mathematical first modal frequency.

\subsubsection{Control law $\mathrm{C}$}

Control law $\mathrm{C}$ consists of the following five steps:

1. The control input profile is determined using the predicted modal frequency. Provided that there are no modeling errors, the time responses of the output are predicted using the obtained control input profile, and are treated as the reference trajectories.

2. MPC is applied to make the outputs track the reference trajectories and to limit the bending-moment within an acceptable level before the variation of the bendingmoment and control input becomes approximately constant.

3. The equation of the bending-moment behavior is approximately governed by

$$
\ddot{B_{\mathrm{e}}}+\omega_{1}^{2} B_{\mathrm{e}}=C_{2} b_{2} u .
$$

Let us denote the constant bending-moment and control input as $B_{\mathrm{e} 0}$ and $u_{0}$, respectively. In this case, the modal frequency can be simply estimated as:

$$
\omega_{1} \cong \sqrt{\frac{C_{2} b_{2} u_{0}}{B_{\mathrm{e} 0}}} .
$$

4. $T_{\mathrm{m}}$ denotes the time when the bending-moment becomes zero. The control input profile must be symmetric with respect to $T_{\mathrm{m}}$ to cancel the vibration at the end of maneuver. When a symmetric control profile is used, the final slewing angle becomes twice the slew angle at $T_{\mathrm{m}}$. Therefore, the slewing angle at $T_{\mathrm{m}}$ must be half the desired slewing angle, that is, $\theta_{\mathrm{f}} / 2$. This requirement can be converted as follows:

Provided that the control input is set to be constant from time $T_{0}$ to time $T_{0}+\Delta t$, the control input is then set to zero after $T_{0}+\Delta t$, the bending-moment is constant from time $T_{0}$ to time $T_{0}+\Delta t$. Oscillation then begins after $T_{0}+\Delta t$. If the first modal frequency dominates the bending-moment, $1 / 4$ of the period $\left(\pi / 2 \omega_{1}\right)$ is required for the constant bending-moment to become zero. Therefore, $\Delta t$ must satisfy

$$
\begin{aligned}
\frac{\theta_{\mathrm{f}}}{2}= & \theta_{0}+\frac{\Delta t}{2}\left(2 \dot{\theta}_{0}+C_{1} b_{1} \dot{\theta}_{0} \Delta t\right) \\
& +\frac{\pi}{\omega_{1}}\left(\dot{\theta}_{0}+C_{1} b_{1} u_{0} \Delta t\right),
\end{aligned}
$$

where $\theta_{0}$ and $\dot{\theta}_{0}$ are the slewing angle and angular velocity of the main body at time $T_{0}$, respectively.

5. The reference trajectories for the slewing angle and bending-moment are reset using the measured slewing angle and bending-moment at $T_{0}$, the control input, and the reestimated modal frequency. MPC is then applied to track the regenerated trajectories from $T_{0}$ to $T_{0}+\Delta t$. After the bending-moment reaches zero, MPC is not used and the symmetric control profile with respect to $T_{\mathrm{m}}$ is simply given to the system.

Figure 10 shows the result of control law C, whereby the bending-moment is successfully limited to within the speci-
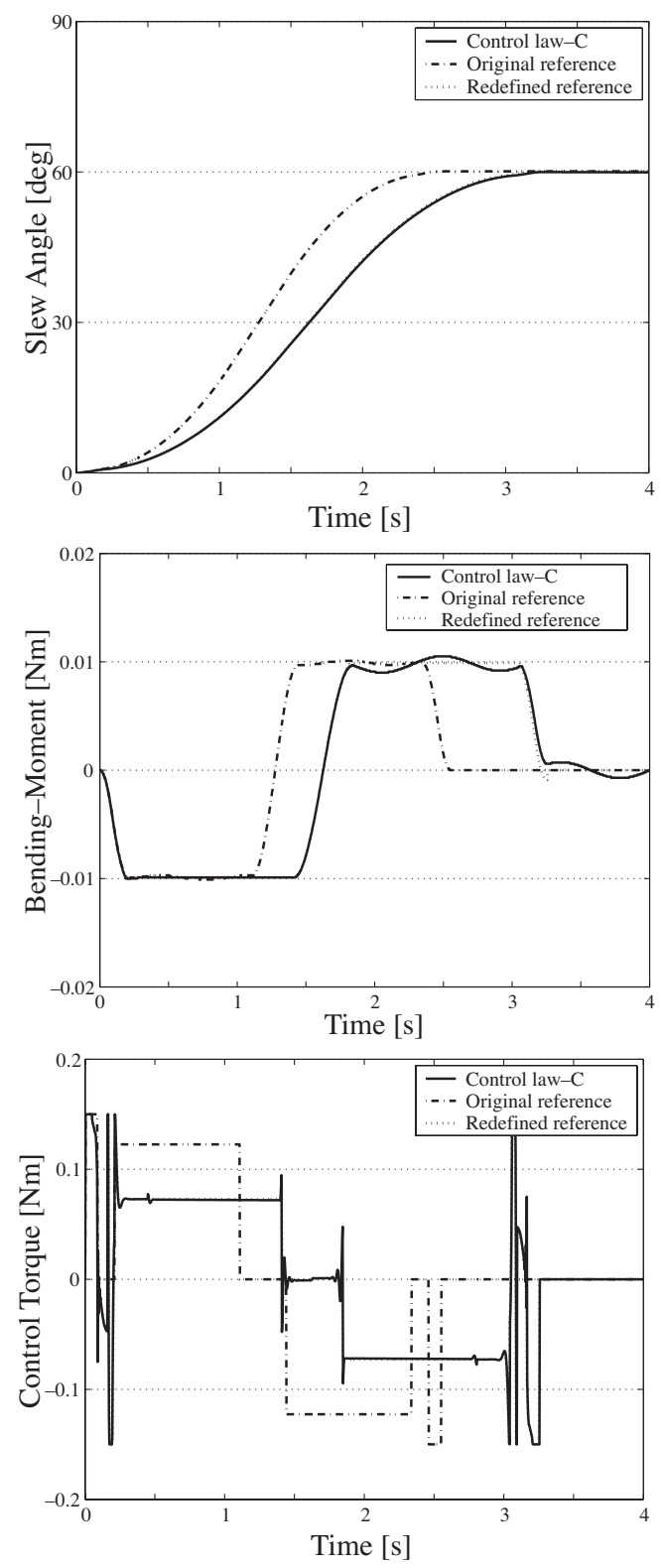

Fig. 10. Results of control law C.

fied range, the residual vibration is suppressed, and the desired slewing angle is achieved, even when the modal frequency is 1.3 times the mathematical modal frequency.

\section{Conclusions}

In this paper, deflection-limiting control in conjunction with model predictive control was studied. Our objectives were to limit the bending-moment at the root of a flexible structure to within an acceptable range, and to achieve a desired slewing angle, and suppress the residual vibration, even in the presence of modeling errors and external disturbances. Three control methods were studied numerically to evaluate performance. The results of the numerical simulations show that control law $\mathrm{C}$, which intends to achieve all the above described objectives has superior performance over control laws A and B, as well as input shaping control methods. 


\section{References}

1) Smith, O. J. M.: Feedback Control Systems, McGraw-Hill Book, Co., Inc., New York, 1958.

2) Singer, N. C. and Seering, W. P.: Preshaping Command Inputs to Reduce System Vibration. J. Dynam. Syst., Meas. Control, 112 (1990), pp. 76-82.

3) Singhose, W. E., Seering, W. P. and Singer, N. C.: Input Shaping for Vibration Reduction with Specified Insensitivity to Modeling Errors, Japan/USA Symposium on Flexible Automation, ASME, New York, N.Y., 1996, pp. 307-313.

4) Singhose, W. E., Derezinski, S. and Singer, N. C.: Extra-insensitive Input Shaping for Controlling Flexible Spacecraft, J. Guid. Control Dynam., 19 (1998), pp. 385-391.

5) Kojima, H., Tanaka, S. and Fujii, A. H.: Minimum Sensitivity Control for Slew Maneuver of a Flexible Space Structure, Trans. Jpn. Soc. Mech. Eng. C, 69 (2003), pp. 396-401.

6) Tzes, A. and Yurkovich, S.: An Adaptive Input Shaping Control Scheme for Suppression in Slewing Flexible Structure, IEEE Trans. Control Syst. Technol., 1 (1993), pp. 114-121.

7) Bodson, M.: An Adaptive Algorithm for the Tuning of Two Input Shaping Methods, Automatica, 34 (1998), pp. 771-776.

8) Stergiopoulos, J. and Tzes, A.: Adaptive Input Shaping for Nonlinear Systems: a Case Study, J. Dynam. Syst., Meas. Control Trans. ASME, 129 (2007), pp. 219-223.

9) Singhose, W., Banerjee, A. and Seering, W.: Slewing Flexible Spacecraft with Deflection-limiting Input Shaping, J. Guid. Control Dynam., 20 (1997), pp. 291-298.
10) Fujii, H., Kojima, H. and Nakajima, N.: Slew Maneuver of a Flexible Space Structure with Constraint on Bending-Moment, J. Guid. Control Dynam., 26 (2003), pp. 259-266.

11) Kojima, H. and Nakajima, N.: Multi-objective Trajectory Optimization by a Hierarchical Gradient Algorithm with Fuzzy Decision Logic-Application to Slew Maneuver Problems of a Flexible Space Structure, T. Jpn. Soc. Aeronaut. Space Sci., 47 (2004), pp. 66-74.

12) Kojima, H. and Singhose, W.: Adaptive Deflection-limiting Control for Slewing Flexible Space Structures, J. Guid. Control Dynam., 30 (2007), pp. 61-67.

13) Kenison, M. and Singhose, W.: Concurrent Design of Input Shaping and Proportional Plus Derivative Feedback Control, J. Dynamic Syst., Meas. Control Trans. ASME, 124 (2002), pp. 398-405.

14) Aldebrez, F. M., Alam, M. S. and Osman Tokhi, M.: Input-Shaping with GA-Tuned Pid for Target Tracking and Vibration Reduction, Proceedings of the 20th IEEE International Symposium on Intelligent Control, and the 13th Mediterranean Conference on Control and Automation, 1999, pp. 485-490.

15) Hu, Q., Shi, P. and Gao, H.: Adaptive Variable Structure and Commanding Shaped Vibration Control of Flexible Spacecraft, J. Guid. Control Dynam., 30 (2007), pp. 804-815.

16) Kojima, H., Fujiki, T. and Fujii, A. H.: $H_{\infty}$ Compensated Minimum Bending Moment Control for Flexible Space Structures, T. Jpn. Soc. Aeronaut. Space Sci., 49 (2007), pp. 254-257.

17) Maciejowski, J.: Predictive Control with Constraints, Prentice Hall, New Jersey, USA, 2002. 